Revue de l'Institut des langues et cultures

d'Europe, Amérique, Afrique, Asie et Australie

$21 \mid 2015$

Discours politique et culturel dans la Russie

contemporaine

\title{
La littérature de jeunesse russe contemporaine et le modèle soviétique : L'enfance de Liova et La légende du grand judo de Boris Minaev
}

Russian Contemporary Youth Literature and Soviet Model: Boris Minaev's The Childhood of Liova and The Genius of Judo

\section{Laure Thibonnier}

\section{OpenEdition}

Journals

Édition électronique

URL : http://journals.openedition.org/ilcea/3054

DOI : 10.4000/ilcea.3054

ISSN : 2101-0609

Éditeur

UGA Éditions/Université Grenoble Alpes

Édition imprimée

ISBN : 978-2-84310-294-3

ISSN : 1639-6073

Référence électronique

Laure Thibonnier, "La littérature de jeunesse russe contemporaine et le modèle soviétique : L'enfance de Liova et La légende du grand judo de Boris Minaev », ILCEA [En ligne], 21 | 2015, mis en ligne le 01 février 2015, consulté le 01 mai 2019. URL : http://journals.openedition.org/ilcea/3054 ; DOI : 10.4000/ilcea.3054

Ce document a été généré automatiquement le 1 mai 2019.

(C) ILCEA 


\section{La littérature de jeunesse russe contemporaine et le modèle soviétique : L'enfance de Liova et La légende du grand judo de Boris Minaev}

Russian Contemporary Youth Literature and Soviet Model: Boris Minaev's The Childhood of Liova and The Genius of Judo

Laure Thibonnier

On sait le carcan idéologique qui a pesé sur la littérature soviétique, y compris d'enfance et de jeunesse, une de ses missions étant de contribuer à l'édification de la société nouvelle. Avec la disparition de l'URSS, de nombreux écrivains de jeunesse se détournèrent des genres sérieux pour expérimenter les jeux formels, l'absurde et l'humour noir, à la manière de Grigori Oster dans ses Mauvais conseils [ВРЕДНЫЕ СОВЕТЫ] dès 1990 (АРЗАМАСЦЕВА, 2012 : 470, 476). Pour autant, comme la spécialiste de littérature de jeunesse Irina Arzamastseva le souligne, dans la deuxième moitié des années 1980 et le début de la période post-soviétique, la société dans son ensemble, et la littérature de jeunesse en particulier, ont dû relever le défi de la représentation et de l'interprétation du passé soviétique : «Trois questions principales se posaient à la société. Premièrement, l'attitude envers l'URSS et la culture du passé soviétique ${ }^{1}$.» (АРЗАМАСЦЕВA, 2012 : 469)

2 Cette problématique s'est reflétée dans les diverses tentatives de démystification ou, au contraire, de défense de certaines figures symboliques de la littérature de jeunesse soviétique, qu'il s'agisse de personnages comme Pavlik Morozov (voir les positions divergentes de Iouri Droujnikov dans Le délateur 001, ou l'Ascension de Pavlik Morozov [ ДОНОСЧИК 001, ИЛИ ВОЗНЕСЕНИЕ ПАВЛИКА МОРОЗОВА, 1987], avant même la disparition de l'URSS, et de Vladislav Krapivine dans Le garçon de bronze [БРонзовыЙ МАльчик, 1992]) ou d'auteurs, tels Arkadi Gaïdar (avec, par exemple, la polémique entre Boris Kamov, dans 
Arkadi Gaïdar: une cible pour les killers de la presse [АРКАДИЙ ГАЙДАР. МИШЕНЬ ДЛЯ ЖУРНАЛЬНЫХ КИЛЛЕРОВ, 2009] et Vladimir Solooukhine, dans Le lac salé [СОЛЁНОЕ ОЗЕРО, 1994]). D'autres écrivains se tournèrent vers la prose historique, comme Edouard Ouspenski et Boris Akounine, qui traitèrent tous deux du Temps des troubles, respectivement dans Le véritable deuxième faux Dimitri [ЛЖЕДМИТРИЙ ВТОРОЙ, НАСТОЯЩИЙ, 1999] et Livre pour enfants [ДЕТСКАЯ КНИГА, 2005]. Récemment, la science-fiction s'est emparée du sujet du retour d'enfants du $\mathrm{XXI}^{\mathrm{e}}$ siècle dans le passé soviétique (Tamara Krioukova, Révision [ПОВТОРЕНИЕ ПРОЙДЕННОГО, 2014], Evgenia Pasternak et Andreї Jvalevski, Toutes les époques sont bonnes [ВРЕМЯ ВСЕГДА ХОРОШЕЕ, 2009]). D’autres ouvrages s'inscrivent, eux, dans la tradition de la prose psychologisante, et témoignent d'une inspiration autobiographique plus ou moins marquée où les auteurs partagent avec leurs jeunes lecteurs leurs propres souvenirs d'enfance :

Le personnel, l'autobiographique est représenté en lien avec le général, le générationnel. À travers la personnalité et les gestes du héros, à l'aide des procédés des types littéraires et de l'individualisation, est brossé le portrait d'une génération, telle qu'elle est vue avec un certain recul temporel ${ }^{2}$. (АРЗАМАСЦЕВВ, $2012: 482$ )

C'est notamment le cas des deux recueils de récits qui ont fait connaitre Boris Minaev, L'enfance de Liova [ДЕТство лёвы, 2001] et La légende du grand judo [ГЕниЙ Дзюдо, 2005]. Boris Minaev naît à Moscou en 1959, dans une famille plus proche du monde de l'industrie que de celui de la littérature : son père dirige un grand complexe textile, sa mère est spécialiste du traitement des fibres chimiques. Le jeune Boris, lui, se destine au journalisme, persuadé que c'est la profession qui, à l'époque, lui laissera le plus de liberté. Son diplôme en poche, il débute dans un journal destiné à la jeunesse : la Komsomolskaïa Pravda, qui le missionne en 1982 dans un séminaire de littérature de jeunesse d'où émergera la célèbre Poule noire [чЁРНАЯ КУРИЦА]. Ce groupe d'écrivains de littérature de jeunesse apparaît au moment de la perestroïka et réunit plusieurs des meilleurs écrivains de jeunesse contemporains (Marina Moskvina, Iouri Netchiporenko, Sergeï Sedov, Tim Sobakine, Boris Minaev...). Dans un premier temps, Minaev poursuit sa carrière journalistique toujours en lien avec la jeunesse puis, après le changement de régime, on le retrouve dans des revues destinées aux adultes (БиБлиогид, 2002; элЕКтРонныЕ ПАМПАСЫ, 2010).

4 Toutes ces années, Minaev rédige ses souvenirs d'enfance, qui donneront naissance à L'enfance de Liova. Leur manuscrit lui permet d'entrer à l'Union des écrivains; le livre paraît en 2001 et obtient la $2^{\mathrm{e}}$ place du prix « Le rêve le plus cher » [" ЗАВЕTHAЯ MEYTA »] en 2006. En 2005 parait la suite de ce recueil, intitulée La légende du grand judo, elle aussi nominée pour des prix littéraires russes importants. Situés dans la deuxième moitié des années 1960 et au début des années 1970, à l'époque de la stagnation brejniévienne, ces ouvrages s'inscrivent si bien dans la vague du "rétro soviétique ${ }^{3}$ " qu'on a pu les comparer à des classiques de la littérature de jeunesse soviétique tels que Les récits de Denis [ДЕНИСКИНЫ РАССКАЗЫ] de Viktor Dragounski, Le chemin s'en va vers le lointain [ДОРОГА уходИТ В ДАЛЬ] d'Alexandra Brouchtein et Vitia Maleev à l'école et à la maison [ВИТя МАЛЕЕВ В школЕ И домА] de Nikolaï Nosov (Bykov, 2011 : 6). Ils traitent tous deux de l'enfance de Liova, double littéraire de Minaev en qui les dernières générations grandies à l'époque soviétique se sont reconnues.

Ces deux recueils mêlent donc l'inspiration autobiographique à une évocation historique particulièrement soignée, et ont un double destinataire: le lecteur adolescent du début $\mathrm{du} \mathrm{XXI}^{\mathrm{e}}$ siècle, souvent ignorant ou peu au fait des réalités soviétiques, et le lecteur adulte 
ayant eu une enfance plus ou moins similaire à celle de Minaev. Cette double nature de L'enfance de Liova et de La légende du judo appelle plusieurs interrogations. Dans un premier temps, on peut questionner le modèle de la vie soviétique proposé par Minaev à des lecteurs adolescents : qu'a-t-il choisi d'en montrer à la jeune génération actuelle, et quelle image cette dernière en aura-t-elle donc? L'écriture autobiographique peut-elle dépasser les clivages idéologiques et laisser la place à un discours qui ne soit ni antisoviétique, ni pro-soviétique? On peut également se demander en quoi ces livres retiendraient l'attention du jeune lecteur, puisqu'ils parlent d'une époque qui, avec la fin de l'URSS et l'avènement du capitalisme, pourrait un peu rapidement être tenue pour révolue et obsolète. Si le lecteur adulte peut s'identifier à Liova parce qu'il retrouve son enfance dans celle de ce dernier, quelle identification peut-il bien y avoir pour un adolescent?

\section{L'esprit du temps}

Les récits de Minaev s'inscrivent dans un chronotope bien précis : la deuxième moitié des années 1960 et le début des années 1970 en URSS, et plus précisément dans le quartier de Presnia $^{4}$, à Moscou, où vit Liova et où Minaev a lui-même grandi. C'est un quartier soviétique à souhait : une insurrection ouvrière y éclate dès 1905 , d'où son nom à l'époque soviétique, Presnia la Rouge [КРАСНАЯ ПРЕСНЯ]. Pour Minaev, dans ce quartier industriel ${ }^{5}$ , tout parlait de la révolution bolchevique : «[...] les rues, les écoles, les maisons de la culture, les gares, les stations de métro [...], tout cela portait le nom de révolutionnaires et de bolcheviques ${ }^{6}$.» (элЕКтронныЕ ПАМПАсЫ, 2010) Liova habite rue du rempart des trois collines, puis avenue Presnia la Rouge ; il fréquente assidûment la rue Zamorionov, du nom d'un révolutionnaire bolchevique ${ }^{7}$. Le quartier compte trois stations de métro au nom éloquent : Barricades, Presnia la Rouge et 1905 (cette dernière n'existait pas encore à l'époque où se situe le récit). Pour autant, ce qui prédomine dans la description de Minaev, ce sont les petites rues et les cours: tranquilles, retirées, pleines de terre, d'arbres, d'herbes, avec des vestiges de maisons en bois ${ }^{8}$, bien éloignées de l'image de mégapole associée à Moscou.

7 Autre élément suscitant la nostalgie : les objets du quotidien, divers et variés, parmi lesquels on relèvera le filet à provisions appelé avos'ka (Minaev, 2011a: 46), les caoutchoucs qu'on met sur ses chaussures pour les protéger de la pluie et de la boue (47), les insignes de pionnier, komsomol ou autres (234). Minaev raconte l'apparition du téléphone individuel (63) et de la télévision (38-39). Le quotidien officieux apparaitt également : dans un pays où le sexe était réputé ne pas exister, un ami montre à Liova des cartes postales pornographiques (Minaev, 2011b:388-390), un autre camarade, une copie à la main du Monopoly, jeu éminemment capitaliste (195-199).

8 Les références à la culture soviétique sont également nombreuses. Minaev évoque les chansons : les parents de Liova apprécient Alexandre Vertinski (Minaev, 2011b : 307), une star de la romance soviétique après son retour d'émigration en 1943, alors que leur fils préfère des groupes plus récents comme « Les joyeux garçons » [« ВЕСЁЛЫЕ РЕБЯТА »]. Les classiques évoqués retracent l'histoire de l'Union soviétique: Au loin, de l'autre côté du fleuve... [ТАм ВДАЛИ ЗА РЕКОЙ...] (Minaev, 2011a: 11) narre la défaite des troupes de Boudionny pendant la guerre civile. Le châle bleu [синиЙ ПЛАТОчЕк] (90) était un succès pendant la Seconde Guerre mondiale, période à laquelle renvoie également la chanson Échangeons à l'aveugle... [МАХНЁМ, НЕ ГЛЯДя...] (79), interprétée par une autre célébrité, 
Mark Bernes. Cette dernière date de 1967, comme La couronne du Danube [ВЕНОК ДУНАЯ ] (12), qui évoque l'amitié entre les pays du camp socialiste et était interprétée par la chanteuse polonaise Edita Piekha. Certains de ces titres font partie de la bande-son de classiques du cinéma soviétique, eux aussi consacrés à des thématiques historiques: on peut entendre Au loin, de l'autre côté du fleuve... dans Et l'acier fut trempé [КАК ЗАКАЛЯЛАСЬ СТАль, 1973 pour la série télévisée, 1975 pour le film], tiré du livre autobiographique de Nikolaï Ostrovski consacré à la guerre civile et aux années suivantes. Échangeons à l'aveugle... a été utilisé dans Le bouclier et l'épée [щит и мЕч], film de 1968 sur la Seconde Guerre mondiale.

Le cinéma est d'ailleurs largement présent dans les souvenirs de Minaev. Tous les dimanches, le père de Liova lui laisse un rouble pour la séance du matin. Le jeune garçon voit les classiques soviétiques, comme Libération [освовождение, 1968-1972] (Minaev, 2011a: 94), et les films des pays satellites, surtout ceux où a tourné Goïko Mikitch (Minaev, 2011b : 337-348), un culturiste yougoslave jouant l'Indien dans la plupart des films de cow-boys et d'Indiens produits en RDA. Quant au titre du $2^{\mathrm{e}}$ recueil, il renvoie au film de Kurosawa La légende du grand judo, dont le titre a été traduit en russe par Le génie du judo [Гений дзюдо]. Tourné en 1943, il a fait l'objet, en 1965, d'un remake par Sanshiro Sugata, sous la tutelle du maitre ; c'est probablement cette version qu'ont vue Liova et Minaev. Minaev évoque également les premières émissions de télévision: Bonne nuit les petits [споКойной нОчИ, МАлыши!] et, pour les plus grands, KVN, un jeu intellectuel humoristique dans lequel s'affrontaient des équipes amateurs, ou encore Le cabaret des 13 chaises, avec, cette fois-ci, des humoristes et des acteurs professionnels (Minaev, 2011a : 40).

10 Cette évocation nostalgique du quotidien soviétique est loin de l'image d'Épinal : il ne s'agit pas pour Minaev de regretter un âge d'or perdu, bien au contraire. Les étals des magasins sont irrégulièrement fournis 9 . Le cinéma « Barricades » est mal famé, Liova s'y fait racketter (Minaev, 2011a : 352-354), il manque tomber entre les mains d'un maniaque (360-361). La voisine se fait régulièrement battre comme plâtre par son mari alcoolique (Minaev, 2011b : 296-302). Un camarade de classe, fils de KGBiste, fait commerce d'images pornographiques (388-390, 392). La sentinelle en faction devant un bâtiment de l'Armée rouge en face de l'immeuble de Liova monnaye le droit de contempler son pistolet (Minaev, 2011a : 224-235). Dans une société proclamée égalitaire et homogène, la mère de Liova méprise les ouvriers parmi lesquels elle vit (63-64). La nounou de son petit frère est une ci-devant (Minaev, 2011b : 61-75). Son oncle, de retour de camp, a interdiction de résider à Moscou, et rencontre secrètement sa famille dans l'appartement de Liova (Minaev, 2011a : 102-121). Dans cette société qui célèbre l'héroïsme des soldats pendant la Seconde Guerre mondiale, chaque famille compte des disparus et des estropiés, et on parle peu des épreuves que chacun a traversées (85-95): autant le sexe semble être le tabou autour duquel se construit La légende du judo, autant l'impact quotidien d'une guerre pourtant terminée (et pas son image officielle) semble être celui de L'enfance de Liova. Bien qu'imprégnée de nostalgie, cette évocation de l'URSS des années 1960-1970 ne verse donc pas dans l'idéalisation.

11 On peut toutefois se demander ce que, au-delà des clichés du quotidien soviétique, les adolescents d'aujourd'hui comprennent au texte de Minaev. Le récit consacré à l'oncle sorti de camp, déjà évoqué ci-dessus, est à leur portée, car, Liova ne comprenant pas la situation de son oncle, ses parents la lui expliquent. Le jeune lecteur contemporain découvre ainsi tout un pan d'une réalité soviétique parfois occultée ou schématisée. Mais 
un autre exemple en dit long sur les changements qu'a connus la Russie. Tout un chapitre est consacré aux aventures de Liova et sa mère dans le centre commercial (l'univermag) de leur quartier. Rien d'exceptionnel pour un adolescent du début du xxI ${ }^{\mathrm{e}}$ siècle, mais Liova, lui, est comme transporté dans un monde de rêve :

Je n'oublierai jamais ce que j'ai ressenti la première fois que j'ai pénétré dans ce magasin feérique. On y jouait de la musique, diverses marchandises étaient exposées sur des rayons de diverses tailles, les gens passaient lentement et cérémonieusement d'un comptoir à l'autre, et tout au centre de la salle se tenait un milicien $^{10}$. (Minaev, 2011b : 28-29)

12 La production officielle ne plaisant pas à Liova, sa veste (polonaise, une rareté !) sera achetée à la sauvette dans les toilettes. Aussi, lorsque les espiègleries de Liova provoquent l'apparition d'un milicien, sa mère cache son effroi en attaquant la cliente prête à la dénoncer (Minaev, 2011b : 28-45). Dans cet épisode, il faut tellement lire entre les lignes pour saisir les motifs implicites de certains actes et paroles des protagonistes qu'un enfant d'aujourd'hui ne peut pas tout saisir. Pour autant, les livres de Minaev ne s'adressent pas qu'à des adultes nostalgiques. En le lisant, l'enfant russe du xxI siècle doit simplement, par endroits, faire la même chose qu'un étudiant en séjour linguistique dans un pays étranger: deviner d'après le contexte, comme le suggère Minaev lui-même lorsqu'il affirme qu'« il faut écrire de façon que tout soit clair d'après le contexte ${ }^{11}$ » (эxO москвы, 2011). Pour lui, au-delà de la peinture d'une époque somme toute conjoncturelle (il décrit cette période parce que le hasard a voulu qu'il y naisse), « le plus important, ce sont [...] les situations humaines ${ }^{12}$ » (эхо москвы, 2011) - situations qu'on devine inhérentes à la condition humaine et donc propices à l'apparition d'un champ commun entre les lecteurs adolescent et adulte.

\section{L'enfance intemporelle}

13 Derrière l'esprit du temps, il y a en effet dans L'enfance de Liova et La légende du judo quelque chose d'éternel, qui en appelle à des invariants qui structureraient immanquablement l'expérience humaine et pourraient servir de points de repère et d'identification aux jeunes lecteurs. Ces invariants sont tous liés à l'expérience du temps vécu : ce sont les étapes que l'enfant franchit pour devenir adulte, mais c'est aussi le regard porté par l'adulte se retournant sur son passé. Minaev décrit ainsi à la fois ce que son jeune lecteur a déjà vécu, ce qu'il est en train de vivre, mais aussi ce qu'il vivra arrivé à l'âge adulte.

Certaines choses ne changeront jamais dans l'enfance. Tous les enfants prendront plaisir aux mêmes expériences : étudier les propriétés de l'eau tombant du balcon, au grand dam des passants (Minaev, 2011a : 139-152); construire une digue dans la cour pour faire une énorme flaque, puis tout détruire (288-302). Ils partageront tous les mêmes rêveries: jouer avec un rayon de soleil passant entre les rideaux de bon matin, s'imaginer sa chambre à hauteur d'insecte (79-80). Ils connaîtront tous les bonheurs et les affres de la socialisation: les amis qu'on suivrait jusqu'au bout du monde, même si on ne les comprend pas toujours; les démêlés avec la petite terreur de l'école ; l'établissement des relations entre filles et garçons (Minaev, 2011b). Une chose peut-être distinguera l'enfance de la génération de Minaev et celle de l'adolescent du xxI siècle : la liberté des enfants à l'époque, qui ne semble pas avoir survécu aux changements, sauf peut-être à la campagne ou dans les petites villes. Les enfants passaient leur temps dehors, dans les 
cours, les adultes ne les appelant que pour déjeuner et lorsque la nuit tombait. Ils vivaient leur propre vie, faisaient leurs découvertes (de la nature, des autres) à l'écart de leurs parents.

Le jeune lecteur contemporain se reconnaîtra également dans les peurs de Liova. Lui aussi a éprouvé l'angoisse de la séparation, d'abord tout petit, lorsque sa maman s'éloignait ${ }^{13}$, puis plus grand, lorsque ses parents le laissaient seul à la maison et que, soudain, il réalisait que, sans eux, il n'existait pas ${ }^{14}$. L'idée de la mort future de leurs parents s'est un jour nettement imposée à tous deux. Attentif à chaque mouvement de l'âme de son jeune héros, Minaev décrit cette brutale prise de conscience avec une rare finesse psychologique; de ce point de vue, son écriture, à la fois réaliste et intimiste, psychologisante, rappelle fortement le style de Gaïdar, un de ses modèles avoués en littérature ${ }^{15}$. Allongé dans son lit, Liova remarque des fissures sur le plafond; elles forment un visage, qui s'anime bientôt :

« Ton père et ta mère mourront », disait tristement le Visage.

Désespéré, je me couchais sur l'autre côté et me cachais à nouveau sous la couverture. Je jetais un œil par la fente, mais la bande de lumière avait disparu, seul demeurait le parquet fissuré et taché. Et mes vieilles pantoufles juste sous mon nez. « Non, pensai-je. Ca arrivera dans longtemps, lorsque je serai moi-même âgé. J'aurai cinquante ans. Tout sera différent, la terre, le ciel... Les souhaits des gens se réaliseront. Il n'y aura plus de mort. »

Je m'allongeais à nouveau sur le dos. «Je t’ai cloué le bec, hein? dis-je au Visage. La mort n'existera plus!»

« Mais la bande de lumière a disparu, répondit le Visage. Elle n'est plus là. Elle était là, et elle s'est évanouie. Évaporée comme une flaque d'eau. C'est la même chose pour la vie. Au début, elle existe. Ta mère se lèvera et préparera des œufs au plat. Ton père ouvrira la porte du balcon et soulèvera ses poids. À la radio, on donnera l'émission "Bonjour !". Mais ce n'est qu'une bande de lumière. Et elle prendra fin. Pfff, gamin ${ }^{16}$ ! (Minaev, 2011a : 80-81)

Peur de la séparation, pressentiment de la sexualité (Minaev, 2011b:20-21), peur de grandir ${ }^{17} \ldots$ : ce mélange doux-amer de joies et de peurs que restitue Minaev, c'est la saveur unique de l'enfance. Il nous montre Liova franchir les étapes par lesquelles passe tout enfant sur le chemin qui mène à l'âge adulte. Ce processus éternel et universel représente justement ce champ commun qui permet l'identification des lecteurs adulte et adolescent à Liova, mais aussi leur identification mutuelle : le lecteur adulte se souvient de sa propre enfance, le lecteur adolescent mesure le chemin parcouru par les adultes qui l'entourent, et qui lui reste encore en partie à parcourir. Ainsi Minaev ne propose-t-il pas seulement à ses lecteurs un modèle de la vie soviétique qui a été la sienne, mais aussi, plus généralement, un modèle du cheminement de l'enfance à l'âge adulte.

\section{Le temps qui passe}

Or, ce cheminement s'effectue dans le temps : finalement, au-delà des années 1960-1970, au-delà de l'enfance, le véritable sujet de Minaev, c'est le temps. Il le formule nettement dans son troisième livre, Le psychologue, ou l'erreur du docteur Levin [ПсихолоГ, Или ошиБКА доктоРА ЛЕвИНА], où l'on retrouve Liova adulte: «Réfléchissons un peu avec Liova au temps. Le temps, c'était le sujet de réflexion que Liova préférait. De ce temps-là, à cette époque... Où allait le temps, d'où venait-il ${ }^{18}$ ? (Minaev, 2007) Minaev invite son lecteur à faire l'expérience du temps qui passe, d'une époque historique à l'autre, d'un âge individuel à un autre, du passé au présent. Ce regard réflexif de celui qui se retourne sur 
son passé est cette fois commun à l'auteur et au lecteur adulte, et Minaev propose au lecteur adolescent de s'y initier par la lecture de L'enfance de Liova et de La légende du grand judo.

Ces recueils représentent un va-et-vient incessant entre le passé et le présent. Par exemple, les fins de récits représentent souvent l'écho présent de pensées ou d'événements passés, comme ici ${ }^{19}$ :

Depuis lors, j'ai souvent été chez le dentiste. Ils doivent avoir un lien de télépathie, parce que tous sans exception m'ont regardé d'un air soupçonneux et m'ont dit de bien me tenir, de ne pas soupirer ni m'agiter. Mais maintenant, il n'y a plus personne pour m'acheter de lapin.

Pourtant, étrangement, chaque fois que j'éprouve de la douleur (n'importe quelle douleur, pas forcément liée aux dents), je l'attends. Je l'attends, alors même que je sais que cet espoir est vain.

Lapin, m'entends-tu? (Minaev, 2011a : 27)

Tant et si bien que les frontières entre passé et présent se brouillent : le passé, pourtant révolu, continue à exister dans le présent à travers la conscience de celui qui l'a vécu et l'évoque. Cet entrelacs des époques est particulièrement sensible dans le premier récit de L'enfance de Liova, qui, à cet égard, donne une clé de lecture des deux recueils. On y voit la mère de Liova lui inventer un conte, lui réciter le début d'un célèbre poème de Pouchkine consacré à Oleg le Sage, un des premiers princes de la Rus', puis lui chanter un chant communiste (Minaev, 2011a: 11). Toutes ces images se mélangent dans l'esprit du petit garçon :

Je voyais nettement une centaine de jeunes soldats de l'Armée rouge, et Oleg le Sage dans sa veste de commandant en cuir à leur tête, et le sorcier qui le mettait en joue de son fusil à lunette, et beaucoup d'autres choses, le cheval moreau, sa crinière penchée sur Oleg, à moins qu'il ne se fût agi de ce garde rouge, le plus jeune, le plus beau ${ }^{20}$... (Minaev, 2011a : 11-12)

Ces personnages d'époques différentes sont appréhendés comme appartenant à la même réalité, comme concomittants : ils peuplent le monde intérieur de Liova et, à ce titre, semblent toujours vivants et présents.

Cet entrelacs du passé et du présent renvoie à ce que Paul Ricœur a défini comme "l'énigme du souvenir en tant que présence de l'absent antérieurement rencontré " (Ricœur, $2000: 47$ ) - autrement dit, à la mémoire, notion connexe de celle du temps. Comme chez lui, la mémoire connaît chez Minaev une triple attribution : soi-même, les proches et les collectifs (Ricœur, 2000 : 152-163). La figure de Liova est en effet multiple. Dans le premier recueil, le narrateur parlant à la première personne du singulier et n'étant désigné par son prénom qu'à la page 37, il est facile d'identifier Liova et Minaev. Or, dans sa postface, l'auteur souligne que Liova tient plutôt du héros collectif, empruntant de nombreux traits autant à Minaev qu'à ses proches :

Mais plus je les [les récits - LT] écrivais, plus j'oubliais ce qui m'était arrivé et ce qui était arrivé à mon frère Micha, les impressions que j'avais eues, ce qui m'avait été rapporté par des camarades dans notre cour. [...] soudain, je compris que j'avais complètement oublié et mélangé ce qui avait réellement eu lieu et ce qu'on m'avait raconté ou que j'avais inventé. Alors je décidai de parler de Liova. Liova est mon ami, il existe réellement. Mais je décidai de le laisser se promener à ma place sur les pages de ce livre ${ }^{21}$. (Minaev, 2011a : 401-403)

Bien loin de nuire à l'ambition véritative de la mémoire, cette triple attribution permet à Minaev de créer un personnage qui sorte du cadre de la mémoire individuelle, forcément limitée, partielle et partiale, et qui devienne emblématique de toute une génération. 
21 autour de lieux de mémoire qui, comme chez Pierre Nora, sont aussi bien symboliques (voir tout le champ de la culture soviétique rappelé précédemment) que topographiques. Très souvent, lorsqu'il évoque une anecdote du passé, Minaev la relie au lieu dans lequel elle s'est déroulée. Le procédé est poussé à l'extrême dans La légende du grand judo, où Minaev soit situe ce lieu par rapport à plusieurs points de repère significatifs (un magasin, un kiosque, une station de métro...), soit nomme l'édifice qui s'y élève aujourd'hui. Cette stratégie à la fois révèle la propension du souvenir à surgir à la faveur d'un lieu et renforce le statut réaliste de la représentation faite par Minaev de l'époque soviétique $^{22}$.

Ce procédé est particulièrement saisissant dans ce passage où Minaev évoque le parc Pavlik Morozov :

Si jamais il vous prenait l'envie de venir dans le parc voir la statue, notez bien qu'elle n'existe plus. On l'a déplacée (à mon avis, dans la Maison des pionniers, à moins qu'on ne l'ait tout simplement fondue).

Disparu aussi, le splendide panorama sur la Moscova : cela fait bien longtemps, une quinzaine d'années, que l'énorme Maison blanche l'a bouché. Plus d'inscription « Parc pour enfants Pavlik Morozov ». On n'y vend toujours ni bière, ni glace.

Aujourd'hui, dans le parc, il y a une chapelle en bois. À la mémoire de ceux qui ont perdu la vie par hasard ou bêtise lors des événements de 93. Ce qui fait que, d'une certaine façon, cet endroit a toujours quelque chose à voir avec l'outre-tombe.

Même s'il se trouve encore quelques filles pour y faire de la luge ${ }^{23}$. (Minaev, 2011b : 187-188)

La conclusion de ce récit condense en quelques phrases près de quatre-vingts ans d'histoire, des années 1930 où le jeune Pavlik Morozov dénonça son père comme koulak au début $\mathrm{du} \mathrm{xxi}{ }^{\mathrm{e}}$ siècle auquel renvoie la chapelle, en passant par les événements des années 1991 et 1993 qui établissent une rupture entre l'avant (le passé) soviétique et l'après (le présent) démocratique. Ainsi l'art de la mémoire tel que le professe ici Minaev permet-il d'établir un pont entre les générations et de renouer des liens entre les époques historiques malgré les ruptures politiques, comme le suggère l'image des jeunes filles.

Cette inscription du temps dans l'espace est si importante pour Minaev qu'il la reprend dans Le psychologue... :

J'adore les cours. Une cour, ce n'est ni la maison, ni la rue. C'est comme un état intermédiaire de l'être humain. Liova aimait beaucoup tout ce qui était dans l'intervalle. Simplement se promener pendant une demi-heure. Passer un moment allongé sur le divan, juste comme ça. Tous ses amis le tenaient pour un paresseux indécrottable, pathologique ${ }^{24}$. (Minaev, 2007)

Le lieu (la cour, le divan) est corrélé au laps de temps qui y est passé (une demi-heure, un moment), et cette corrélation renvoie à un état d'âme (l'« état intermédiaire de l'être humain») défini comme "intervalle». Pour Liova comme pour Minaev, le défi est d'exister dans l'intervalle: entre l'époque soviétique et la Russie post-soviétique, entre l'enfance et l'âge adulte, entre les souvenirs et la réalité, entre le passé et le présent, mais aussi entre soi (la citation commence par «je ») et autrui (le «je » devient rapidement «Liova », dont on sait désormais qu'il n'est pas seulement Minaev). Cet intervalle, c'est aussi le laps de temps qui s'écoule entre le moment où a lieu une chose et celui où on s'en souvient. Or, ce laps de temps peut être ressenti sur le mode de la nostalgie (Ricœur, 2000 : 49). C'est précisément cette nostalgie du temps qui passe et qui ne reviendra pas, tout en étant encore présent par l'intermédiaire de la mémoire, qui imprègne les recueils

ILCEA, 21 | 2015 
de Minaev, et seule une lecture hâtive pourrait conduire à la confondre avec la nostalgie de l'époque soviétique.

\section{BIBLIOGRAPHIE}

100 новыХ Книг дЛЯ ДЕТЕЙ и ЮношЕСтвА (2007), <www.grand-fair.ru/files/GRAND2-V.pdf> (17 juin 2014).

АРЗАМАСЦЕВА ИРИНА (2012), ДЕТСКАЯ ЛИТЕРАТУРА. УЧЕБНИК, МоsсоU : ИЗД. ЦЕНТР « АКАДЕМИЯ ». БИБЛИогИД (2002), МИНАЕВ БОРИС ДОРИАНОВИЧ. НЕОБХОДИМЫЕ ВОПРОСЫ, <http://bibliogid.ru/ pisateli/pisateli-o-sebe/654-minaev-boris-dorianovich? highlight=WyJcdTA0M2NcdTA0MzhcdTA0M2RcdTA0MzBcdTA0MzVcdTA0MzIiXQ> (17 juin 2014). БЫКОВ ДМИТРИЙ (2011), « ОБ ЭТОЙ КНИГЕ », МИНАЕВ БОРИС, ДЕТСТВО ЛЁВЫ (6-7), Moscou : КОМПАСГИД.

ЭХО МОСКВЫ (2011), ДЕТСКАЯ ПЛОЩАДКА С ПАПАШЕЙ БУНТМАНОМ: ПИСАТЕЛЬ БОРИС МИНАЕВ ПРЕДСТАВЛЯЕТ СВОЮ НОВУЮ КНИГУ «ЧУЖИЕ РЕБЯТА», <www.echo.msk.ru/programs/buntmankid/772521-echo/> (18 juin 2014).

ЭЛЕКТРОННЫЕ ПАМПАСЫ (2010), БОРИС МИНАЕВ, <www.epampa.narod.ru/minaev/index.html> (16 juin 2014).

МИНАЕВ БОРИС (2007), « ПСИХОЛОГ, ИЛИ ОШИБКА ДОКТОРА ЛЕВИНА », ОКТЯБРЬ, 11, <http:// magazines.russ.ru/october/2007/11/mi1.html> (18 juin 2014).

МИНАЕв БОРИС (2011a), ДЕТСТВО ЛЁВЫ, Moscou : КОМПАСГИД.

МИНАЕВ БОРИС (2011b), ГЕНИЙ ДЗЮДО, Moscou : КОМПАСГИД.

RICœUR Paul (2000), La mémoire, l'histoire, l'oubli, Paris : Seuil.

\section{NOTES}

1. «ГЛАВНЫХ ВОПРОСОВ, ПОСТАВЛЕННЫХ ПЕРЕД ОБЩЕСТВЕННЫМ СОЗНАНИЕМ, БЫЛО ТРИ. ВО-ПЕРВЫХ, ОТНОШЕНИЕ К СССР И КУЛЬТУРЕ СОВЕТСКОГО ПРОШЛОГО. »

2. «ЛИЧНОЕ, АВТОБИОГРАФИЧЕСКОЕ ИЗОБРАЖАЕТСЯ В ЕДИНСТВЕ С ОБЩИМ, ПОКОЛЕНЧЕСКИМ. В ЛИЧНОСТИ И ПОСТУПКАХ ГЕРОЯ С ПОМОЩЬЮ ПРИЕМОВ ТИПИЗАЦИИ И ИНДИВИДУАЛИЗАЦИИ ДАН ПОРТРЕТ ПОКОЛЕНИЯ, КАКИМ ОН ВИДИТСЯ НА ЗНАЧИТЕЛЬНОМ ВРЕМЕННОМ УДАЛЕНИИ. »

3. C'est ainsi qu'il est présenté dans le catalogue recensant, chaque année, les 100 nouveaux titres de littérature de jeunesse les plus intéressants (100 новых книг для дЕТЕй и юношЕствА, 2007 : 10).

4. «Puis nous avons déménagé dans un deux-pièces individuel à Presnia. " [« Потом мы ПЕРЕЕХАЛИ В ОТДЕЛЬНУЮ ДВУХКОМНАТНУЮ КВАРТИРУ НА ПРЕСНЕ. »] (МИНАЕВ, 2011а : 19)

5. Aujourd'hui, les industries sont déplacées à l'extérieur de la ville pour être remplacées par le centre d'affaires Moskva-city. 
6. «[...] УЛИЦЫ, ШКОЛЫ, ДОМА КУЛЬТУРЫ, ВОКЗАЛЫ, СТАНЦИИ МЕТРО, ТАНКОВЫЕ ДИВИЗИИ, КОРАБЛИ

— ВСЁ БЫЛО НАЗВАНО В ЧЕСТЬ РЕВОЛЮЦИОНЕРОВ И БОЛЬШЕВИКОВ. »

7. «La cour était calme et vide. Le soleil commençait à disparaitre derrière le toit de l'immeuble $\mathrm{n}^{\circ}$ 16. Au-delà commençait la rue Zamorionov, qui dévalait vers le bas avec ses tramways brinquebalants, puis la place de l'Insurrection. Dans notre rue des Trois remparts, il y avait un square, avec à un bout une statue de Lénine ; à l'autre, on avait récemment installé un ouvrier avec des pavés.» [«ВО ДВОРЕ БЫЛО ТИХО, ПУСТО. СОЛНЦЕ НАЧИНАЛО САДИТЬСЯ НА КРЫШУ ШЕСТНАДЦАТОГО ДОМА. ДАЛЬШЕ НАЧИНАЛАСЬ УЛИЦА ЗАМОРЕНОВА, КРУТО БЕГУЩАЯ ВНИЗ, С ДРЕБЕЗЖАЩИМИ ТРАМВАЯМИ, А ПОТОМ ПЛОЩАДЬ ВОССТАНИЯ. А НА НАШЕМ ТРЕХГОРНОМ ВАЛУ БЫЛ СКВЕР. В ОДНОМ ЕГО КОНЦЕ БЫЛ ПАМЯТНИК ЛЕНИНУ, А В ДРУГОМ НЕДАВНО ПОСТАВИЛИ РАБОЧЕГО С Булыжником. »] (Minaev, 2011a : 85)

8. «Les ruelles calmes de Presnia étaient envahies de maisons en bois laissées à l'abandon, de chats et de feuilles mortes; l'eau souterraine bouillonnait sous les regards de chaussée. Elles étaient désertes. On pouvait marcher lentement, tout à ses pensées, ou bien regarder autour de soi. Les voitures ne s'aventuraient presque jamais dans ces ruelles. » [« В ТИХИХ ПРЕСНЕНСКИХ ПЕРЕУЛКАХ С ПОЛУРАЗВАЛИВШИМИСЯ ДЕРЕВЯННЫМИ ДОМАМИ БЕГАЛИ КОШКИ, НОСИЛИСЬ СУХИЕ ЛИСТЬЯ, В ВОДОПРОВОДНЫХ ЛЮКАХ БУЛЬКАЛА ПОДЗЕМНАЯ ВОДА. НАРОДУ ТУТ НИКОГО НЕ БЫЛО. МОЖНО БЫЛО ИДТИ МЕДЛЕННО, ДУМАТЬ О СВОЕМ ИЛИ ГЛАЗЕТЬ ПО СТОРОНАМ. МАШИНЫ В ПЕРЕУЛКАХ ПОЧТИ НЕ Ездили. »] (Minaev, 2011a: 51-52)

«[...] des cours retirées où poussaient des tilleuls séculaires, entourées de palissades penchées dont il était facile d'ôter les planches, et où l'on trouvait quantité d'objets rouillés, vermoulus et intéressants. » (Minaev, 2011a: 54)

9. «[...] le magasin s'appelle "Fruits et légumes", alors qu'on n'y trouve ni pomme de terre, ni oignon, et seulement des fruits secs. » [“ [...] МАГАЗИН НАЗЫВАЕТСЯ “ОВОЩИ - ФРУКТЫ”, А В НЕМ НИ КАРТОШКИ, НИ ЛУКА, И ФРУКТЫ ОДНИ СУШЕНЫЕ. »] (Minaev, 2011a : 13)

10. « НИКОГДА НЕ ЗАБУДУ ТОГО ЧУВСТВА, С КОТОРЫМ Я ВПЕРВЫЕ ПЕРЕСТУПИЛ ЭТОТ ВОЛШЕБНЫЙ ПОРОГ! В МАГАЗИНЕ ИГРАЛА МУЗЫКА, НА ПОЛКАХ И ПОЛОЧКАХ ВИДНЕЛИСЬ РАЗЛИЧНЫЕ ТОВАРЫ, ОТ ПРИЛАВКА К ПРИЛАВКУ МЕДЛЕННО И ЧИННО ХОДИЛИ ЛЮДИ, А В ЦЕНТРЕ ЗАЛА, В САМОЙ ЕГО СЕРЕДИНЕ, СТОЯЛ МИЛИЦИОНЕР. »

11. « НАДО ПРОСТО ВОТ ТАК НАПИСАТЬ, ЧТОБЫ ВСЁ-ТАКИ БЫЛО ПОНЯТНО ИЗ КОНТЕКСТА ».

12. « ВАЖНЕЕ [...] ЭТИ ЧЕЛОВЕЧЕСКИЕ КАКИЕ-ТО СИТУАЦИИ ».

13. «Mais dès que ma mère s'éloignait, pour payer en caisse, s'installer dans le fauteuil chez le coiffeur, je commençais à m'inquiéter terriblement : et si elle disparaissait? » [« А ВОТ КАК ТОЛЬКО МАМА КУДА-НИБУДЬ ОТХОДИЛА - ПРОБИТЬ ЧЕК В КАССЕ, ИЛИ В ПАРИКМАХЕРСКОЕ КРЕСЛО, Я НАЧИНАЛ УЖАСНО БЕСПОКОИТЬСЯ - ВДРУГ ОНА КУДА-НИБУДЬ ПРОПАДЕТ? »] (Minaev, 2011a : 9)

14. «La douleur devenait insupportable. Je ne pouvais tout simplement pas me passer de papa et maman. Sans eux, je ne pouvais ni m'endormir, ni manger, ni boire - je ne pouvais rien faire. Il était étrange et stupide de faire quoi que ce soit tout seul. Ma peur disparaissait, cette peur enfantine idiote et misérable. Ne restait plus qu'une seule pensée : la vie s'était arrêtée. J'étais comme mort. » [« БОЛЬ СТАНОВИЛАСЬ НЕСТЕРПИМОЙ. Я ПРОСТО НЕ МОГ БЕЗ МАМЫ И ПАПЫ. НЕ МОГ БЕЗ НИХ ЗАСНУТЬ. НЕ МОГ ЕСТЬ И ПИТЬ. НИЧЕГО НЕ МОГ ДЕЛАТЬ. ДЕЛАТЬ ЧТО-НИБУДЬ ОДНОМУ БЫЛО ГЛУПО И НЕЕСТЕСТВЕННО. СТРАХ ИСЧЕЗАЛ. МОЙ ГЛУПЫЙ, ЖАЛКИЙ И ДЕТСКИЙ СТРАХ. ОСТАВАЛАСЬ ТОЛЬКО ОДНА, ВОТ ЭТА ОДНА-ЕДИНСТВЕННАЯ МЫСЛЬ: ЖИЗНЬ ОСТАНОВИЛАСЬ. Я КАК МЕРТВЫЙ. »] (Minaev, 2011a : 76) 15. «Parmi mes [lectures - LT] d'enfance, ceux que je considère comme mes maitres sont Gaïdar, Aleksine, Koval. » [« ИЗ ДЕТСКИХ [ЧТЕНИЙ - ЛТ] СВОИМИ УЧИТЕЛЯМИ СЧИТАЮ ГАЙДАРА, АЛЕКСИНА, ковАля. »] (БиБЛИогид, 2002)

16. “"ТВОИ ПАПА И МАМА УМРУТ”, - ПЕЧАЛЬНО СКАЗАЛО ЛИЦО. Я ОТЧАЯННО ПОВЕРНУЛСЯ НА БОК И СНОВА НАТЯНУЛ ОДЕЯЛО. ПРИНИК К ДЫРОЧКЕ. НО СВЕТЛОЙ ПОЛОСЫ УЖЕ НЕ БЫЛО. БЫЛ ПРОСТО ПАРКЕТ С ТРЕЩИНАМИ И ПЯТНАМИ. И ЛЕЖАЛИ ПОД САМЫМ НОСОМ МОИ СТАРЫЕ ТАПОЧКИ. “НЕТ, - ПОДУМАЛ Я. 
— ЭТО БУДЕТ НЕСКОРО, КОГДА Я И САМ БУДУ СТАРИКОМ. МНЕ БУДЕТ ЛЕТ ПЯТЬДЕСЯТ. ВСЕ БУДЕТ ДРУГИМ - И ЗЕМЛЯ, И НЕБО. ВСЕ ЖЕЛАНИЯ ЛЮДЕЙ БУДУТ ИСПОЛНЯТЬСЯ. НИКАКОЙ СМЕРТИ НЕ БУДЕТ.” Я СНОВА ПОВЕРНУЛСЯ НА СПИНУ. “НУ ЧТО, СЪЕЛ? - СКАЗАЛ Я ЛИЦУ. - НЕ БУДЕТ НИКАКОЙ СМЕРТИ!” “А СВЕТЛАЯ ПОЛОСА ИСЧЕЗЛА, - СКАЗАЛО ЛИЦО. НЕТ ЕЕ. БЫЛА И РАСТВОРИЛАСЬ. ВЫСОХЛА, КАК ЛУЖА. ВОТ ТАК И ЖИЗНЬ. СЕЙЧАС ОНА ЕСТЬ. МАМА ВСТАНЕТ И БУДЕТ ЖАРИТЬ ЯИЧНИЦУ. ПАПА ОТКРОЕТ БАЛКОН И НАЧНЕТ, ПЫХТЯ, ПОДНИМАТЬ ГИРЮ. ПО РАДИО БУДЕТ ПЕРЕДАЧА 'С ДОБРЫМ УТРОМ!'. НО ЭТО ТОЛЬКО СВЕТЛАЯ ПОЛОСА. И ОНА КОНЧИТСЯ. ЭХ ТЫ, МАЛЫШ-ГОЛЫШ!” »

17. "Soudain je compris, que ce n'était pas mes parents que j'avais fui, ni la boulangerie.Ce que j'avais fui, c'était un sentiment oppressant d'angoisse, qui ne m'avait pas lâché d'une semelle depuis le matin, tel un rhume ou le hoquet. C'était lui qui m'avait mis de mauvaise humeur, c'était à cause de lui que j'avais mal partout. Mon angoisse, c'était moi. [...] Et j'eus l'impression que cette angoisse m'accompagnerait toujours. [...] Angoisse à tout propos : et si le soleil s'éteignait? Comment réussir à tout faire? Et si on ne m'aimait plus? Et comment faire pour ne pas avoir l'air ridicule, ne pas être idiot, ne faire de mal à personne ? " [« И ВДРУГ Я ПонЯЛ, что УБЕЖАЛ СЮДА НЕ ОТ РОДИТЕЛЕЙ И НЕ ОТ БУЛОЧНОЙ. Я СБЕЖАЛ СЮДА ОТ ЩЕМЯЩЕГО ЧУВСТВА ТРЕВОГИ. ЭТО ОНО ХОДИЛО ЗА МНОЙ ПО ПЯТАМ, С САМОГО УТРА, КАК НАСМОРК ИЛИ ИКОТА. ЭТО ОНО СДЕЛАЛО МЕНЯ ЗЛЫМ И РАЗБИТЫМ. МОЯ ТРЕВОГА - ЭТО И БЫЛ Я. [...] И МНЕ ПОКАЗАЛОСЬ, ЧТО ЭТА ТРЕВОГА ВСЕГДА БУДЕТ СО МНОЙ. [...] ТРЕВОГА ЗА ВСЕ - ЧТОБЫ НЕ ПОГАСЛО СОЛНЦЕ, ЧТОБЫ ВСЕ УСПЕТЬ. ЧТОБЫ ТЕБЯ НЕ РАЗЛЮБИЛИ... ЧТОБЫ НЕ ПОКАЗАТЬСЯ СМЕШНЫМ, НЕ СТАТЬ ДУРАКОМ, НЕ ДЕЛАТЬ НИКОМУ ПЛОХО.»] ( Minaev, 2011a : 49-50)

18. « ВМЕСТЕ С ЛЕВОЙ ЗАДУМАЕМСЯ О ВРЕМЕНИ. ВРЕМЯ - ЭТО ЛЮБИМАЯ ТЕМА ЛЕВИНЫХ РАЗДУМИЙ. В ТО ВРЕМЯ, В ЭТО ВРЕМЯ... КУДА УХОДИТ ВРЕМЯ, И ОТКУДА ОНО ПРИХОДИТ. »

19. «С ТЕХ ПОР Я МНОГО РАЗ БЫВАЛ У ЗУБНЫХ ВРАЧЕЙ. НАВЕРНОЕ, У НИХ У ВСЕХ ЕСТЬ КАКАЯ-ТО ТЕЛЕПАТИЧЕСКАЯ СВЯЗЬ, ПОТОМУ ЧТО КАЖДЫЙ ВРАЧ ОБЯЗАТЕЛЬНО СМОТРИТ НА МЕНЯ С ПОДОЗРЕНИЕМ И ГОВОРИТ, ЧТОБЫ Я ВЕЛ СЕБЯ НОРМАЛЬНО, НЕ ВЗДЫХАЛ И НЕ ВЗДРАГИВАЛ. ТЕПЕРЬ МНЕ НЕКОМУ КУПИТЬ ЗАЙЦА. НО СТРАННОЕ ДЕЛО, КОГДА МНЕ ПРИХОДИТСЯ ТЕРПЕТЬ БОЛЬ, НЕ ЗУБНУЮ, А ВСЯКУЮ, Я ВСЕГДА ЖДУ ЕГО. ЖДУ, ХОТЯ И ЗНАЮ, ЧТО НАДЕЖДА НАПРАСНА. ТЫ СЛЫШИШЬ, ЗАЯЦ? »

20. « Я ХОРОШО ПРЕДСТАВЛЯЛ СЕБЕ СОТНЮ ЮНЫХ БУДЕННОВЦЕВ, И ВЕЩЕГО ОЛЕГА В КОМАНДИРОВОЧНОЙ ТУЖУРКЕ ВПЕРЕДИ, И ЗЛОГО ВОЛШЕБНИКА, КОТОРЫЙ ЦЕЛИЛСЯ В НЕГО ИЗ ВИНТОВКИ С ОПТИЧЕСКИМ ПРИЦЕЛОМ, И МНОГО РАЗНОГО, А ПОТОМ ЕЩЕ ВОРОНОГО КОНЬКА, КОТОРЫЙ СКЛОНЯЛ ГРИВУ НЕ ТО НАД ОЛЕГОМ, НЕ ТО НАД САМЫМ ЮНЫМ, ОЧЕНЬ КРАСИВЫМ КРАСНОАРМЕЙЦЕМ... »

21. « НО ЧЕМ ДАЛЬШЕ Я ИХ [РАССКАЗЫ - ЛТ] ПИСАЛ, ТЕМ БОЛЬШЕ Я ЗАБЫВАЛ: ЧТО БЫЛО СО МНОЙ, А ЧТО - С МОИМ БРАТОМ МИШКОЙ, ЧТО МНЕ ПОКАЗАЛОСЬ, А ЧТО Я УСЛЫШАЛ ОТ КОГО-ТО ИЗ РЕБЯТ ВО ДВОРЕ. [...] ВДРУГ Я ЯСНО ПОНЯЛ, ЧТО ОКОНЧАТЕЛЬНО ЗАБЫЛ И ПЕРЕПУТАЛ - ЧТО БЫЛО НА САМОМ ДЕЛЕ, А ЧТО МНЕ КТО-ТО РАССКАЗАЛ ИЛИ Я ПРИДУМАЛ. И ТОГДА Я РЕШИЛ, ЧТО БУДУ ПИСАТЬ О ЛЕВЕ. ЛЕВА - МОЙ ДРУГ, КОТОРЫЙ РЕАЛЬНО СУЩЕСТВУЕТ В ЖИЗНИ. НО Я РЕШИЛ, ЧТО ПУСТЬ ОН ВМЕСТО МЕНЯ ГУЛЯЕТ ПО эТоЙ КнИЖКЕ. »

22. "C'est sur la surface de la terre que nous nous souvenons avoir voyagé et visité des sites mémorables. Ainsi les "choses" souvenues sont-elles intrinsèquement associées à des lieux. Et ce n'est pas par mégarde que nous disons de ce qui est advenu qu'il a eu lieu. [...] Les lieux "demeurent" comme des inscriptions, des moments, potentiellement des documents, alors que les souvenirs transmis par la seule voix orale volent comme le font les paroles.» (Ricœur, 2000 : 49)

23. «ЕСЛИ ВЫ ЗАХОТИТЕ ПРИЙТИ В ПАРК И ПОСМОТРЕТЬ НА ПАМЯТНИК, УЧТИТЕ - ЕГО БОЛЬШЕ НЕТ. КУДА-ТО ПЕРЕНЕСЛИ (ПОДОЗРЕВАЮ, ЧТО В ДОМ ПИОНЕРОВ, ХОТЯ, МОЖЕТ, И ПРОСТО ПЕРЕПЛАВИЛИ НА МЕТАЛЛ). НЕТ И ПРЕКРАСНОГО ВИДА НА МОСКВУ-РЕКУ - ЕГО ДАВНО УЖЕ, ЛЕТ ПЯТНАДЦАТЬ НАЗАД, ЗАГОРОДИЛ ГРОМАДНЫЙ БЕЛЫЙ ДОМ. НЕТ И ВЫВЕСКИ: “ДЕТСКИЙ ПАРК ИМЕНИ ПАВЛИКА МОРОЗОВА”. ПИВО И МОРОЖЕНОЕ ЗДЕСЬ ПО-ПРЕЖНЕМУ НЕ ПРОДАЮТ. ТЕПЕРЬ В ПАРКЕ СТОИТ ДЕРЕВЯННАЯ ЧАСОВНЯ. В ПАМЯТЬ О ТЕХ, КТО СЛУЧАЙНО ИЛИ ПО ГЛУПОСТИ ПОГИБ ВО ВРЕМЯ ВОЛНЕНИЙ В 93-М ГОДУ. ТАК ЧТО В 
НЕКОТОРОМ СМЫСЛЕ МЕСТО ОСТАЛОСЬ ЗАГРОБНЫМ. ХОТЯ ПО-ПРЕЖНЕМУ НЕКОТОРЫЕ ДЕВОЧКИ КАТАЮТСЯ ТУТ НА САНКАХ. »

24. « ОБОЖАЮ ДВОРЫ. ДВОР - ЭТО НЕ ДОМ, НЕ УЛИЦА. ЭТО НЕКОЕ ПРОМЕЖУТОЧНОЕ ДЛЯ ЧЕЛОВЕКА СОСТОЯНИЕ. ВСЕ, ЧТО В ПРОМЕЖУТКЕ, ЛЕВА ОЧЕНЬ ЛЮБИЛ. ПРОСТО ПРОЙТИСЬ ПО УЛИЦЕ С ПОЛЧАСА. ПОЛЕЖАТЬ НА ДИВАНЕ НИ С ТОГО, НИ С СЕГО. ВСЕ ЕГО ДРУЗЬЯ СЧИТАЛИ, ЧТО ОН ПАТОЛОГИЧЕСКИЙ, НЕПРОХОДИМЫЙ ЛЕНТЯЙ. »

\section{RÉSUMÉS}

Le présent article est consacré à L'enfance de Liova et à La légende du grand judo de Boris Minaev. S'adressant à un double destinataire adulte et adolescent, l'auteur y explore les notions connexes de temps et de mémoire dans des récits largement autobiographiques. La représentation de l'URSS des années 1960-1970 sert de matière à une réflexion sur le passage de l'enfance à l'âge adulte, mais aussi sur le regard réflexif porté par l'adulte sur son enfance. Ce mélange d'évocation historique et de souvenirs individuels illustre certains mécanismes de formation de la mémoire collective russe.

This article is devoted to The Childhood of Liova and The Genius of Judo by Boris Minaev. Writing for a dual adult and adolescent addressee, the author explores the related concepts of time and memory in largely autobiographical stories. The representation of the USSR in the years 19601970 is used as material for reflection on the passage from childhood to adulthood, but also on the reflective look of the adult back to his childhood. This combination of historical evocation and individual memories illustrates some mechanisms of formation of the Russian collective memory.

\section{INDEX}

Mots-clés : Minaev, Enfance de Liova, La légende du grand judo, littérature de jeunesse russe contemporaine, représentations de l'époque soviétique, mémoire individuelle, mémoire collective

Keywords : Minaev, The Childhood of Liova, The Genius of Judo, Russian contemporary youth literature, representations of the Soviet era, individual memory, collective memory

\section{AUTEUR}

\section{LAURE THIBONNIER}

Université Grenoble Alpes, ILCEA 4 (CESC) 